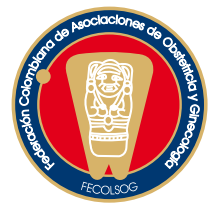

\title{
RELACIÓN ENTRE LAS CARACTERÍSTICAS ESPERMÁTICAS POSCAPACITACIÓN Y LA FERTILIZACIÓN OOCITARIA HUMANA EN LA TÉCNICA DE FERTILIZACIÓN IN VITRO
} \section{The relationship between post-capacitated sperm characteristics and human oocyte fertilization in the in-vitro fertilization technique}

Yamileth Motato M.*, Jaime Saavedra-Saavedra, M.D.**, Martha N. Moyano, M.Sc.***

Recibido: febrero 4/10 - Aceptado: septiembre 7/10

\section{RESUMEN}

Objetivo: establecer la relación entre las características espermáticas poscapacitación y la fertilización de oocitos donados y morfológicamente maduros (estadío MII) en la técnica de fertilización in vitro (FIV).

Metodología: se realizó un estudio de corte transversal entre enero de 2003 y diciembre de 2007 en el Centro de Biomedicina Reproductiva del Valle (Fecundar) en Cali (Colombia). En este estudio, se incluyeron 64 muestras seminales de pacientes que se sometieron a la técnica de FIV y ovodonación. Se comparó la proporción de fertilización de las muestras, asumiendo como apropiada una proporción mayor o igual a $80 \%$. También se utilizó un modelo de regresión logística para determinar las variables que contribuyeron a presentar una mayor probabilidad de fertilización. Resultados: se incluyeron 64 muestras seminales, de un total de 76, que cumplieron con los requisitos

* Bacterióloga y Laboratorista Clínica, Bióloga, GINFEC (Grupo de investigación Fecundar), Cali (Colombia). Correo electrónico: ymotato@yahoo.com

** Especialista en Medicina Reproductiva, Profesor Titular Universidad del Valle, Gintec (Grupo de investigación Fecundar), Cali (Colombia). Correo electrónico: fecundar@fecundar.com

*** Bióloga, Docente Universidad del Valle, Cali (Colombia). Correo electrónico: marmoyano@gmail.com establecidos. En el análisis estadístico se evidenció que la fertilización se afectó por la motilidad poscapacitación tipo a mayor o igual a $25 \%$. El índice de teratozoospermia y los días de abstinencia sexual no afectaron la fertilización oocitaria.

Conclusiones: en los individuos de este estudio la motilidad espermática tipo a poscapacitación mayor a 25\% fue la variable que más influyó en una apropiada fertilización oocitaria en la técnica de FIV.

Palabras claves: características espermáticas, fertilización oocitaria.

\section{SUMMARY}

Objective: establishing the relationship between post-capacitated sperm characteristics and donated fertilized MII (morphologically mature) oocytes when using the in vitro fertilization (IVF) technique.

Methodology: a descriptive cross-sectional study was carried out between January 2003 and December 2007 at the Centro de Biomedicina Reproductiva del Valle (Fecundar) in Cali (Colombia). Seventysix semen samples taken from patients who had undergone IVF and ovodonation were considered in this study; the percentage of fertilization for these samples was compared, assuming fertilization 
higher than or equal to $80 \%$ to be appropriate. A logistic regression model was used for determining the variables contributing towards presenting a greater probability of oocyte fertilization.

Results: sixty-four of the 76 semen samples fulfilled the inclusion criteria. Statistical analysis revealed that fertilization was affected by postcapacitated sperm motility type a higher than or equal to 25\%. The theratozoospermia index (ITZ) and days of sexual abstinence did not affect oocyte fertilization.

Conclusion: post-capacitated sperm motility type a higher than 25\% was the variable that most influenced suitable oocyte fertilization in FIV for the individuals tested in this study.

Key words: spermatic characteristics, oocyte fertilization.

\section{INTRODUCCIÓN}

La infertilidad se define como la incapacidad de la pareja para lograr una gestación. Esta puede ser primaria cuando la pareja nunca ha tenido hijos y secundaria cuando, después de una gestación, existe la imposibilidad de volver a gestar. ${ }^{1}$ Para su tratamiento, se cuenta con técnicas de reproducción asistida (TRA) en las cuales se realiza micromanipulación de espermatozoides, ovocitos y/o embriones. ${ }^{2}$ Entre estas técnicas se encuentran la inyección intracitoplasmática de espermatozoides (ICSI) y la fertilización in vitro (FIV). ${ }^{3}$ Según el registro latinoamericano de reproducción asistida, con esta última se ha reportado una tasa global de embarazo de $27,8 \%{ }^{4}$ por ciclo de tratamiento. La FIV está indicada en infertilidad por factor tubárico, endometriosis severa (estadíos III y IV) y factor masculino con alteraciones mínimas a moderadas en los parámetros seminales: olizoospermia moderada (entre 5 y $10 \times 10^{6}$ espermatozoides $/ \mathrm{mL}$ ) y astenozoospermia moderada [entre 30\% y 50\% de la motilidad progresiva total tipo a (rápida) + tipo b (lenta)] de acuerdo a los valores de referencia establecidos por la Organización Mundial de la Salud (OMS). ${ }^{5}$ La literatura reporta que entre el
$5 \%$ y el 10\% de los casos presentan falla de la fertilización sin evidencia de alteraciones que puedan explicarla. ${ }^{5,6}$

Por otra parte, las alteraciones en la morfología y la vitalidad espermáticas juegan un papel importante en la evaluación de la capacidad fecundante de los espermatozoides y pueden obedecer a las mismas causas (alteraciones en la ultraestructura del flagelo, defectos genéticos, presencia de anticuerpos antiespermáticos, varicocele e infecciones de las vías seminales) que provocan astenozoospermia, por lo que el criterio morfológico estricto se relaciona significativamente con el éxito de la FIV y se considera un índice predictivo de fertilidad. ${ }^{7}$ Un estudio realizado por Kruger et ál. ${ }^{8}$ reveló que la tasa de fertilización fue de 7,6\% en el grupo de parejas que presentó menos de 4\% de espermatozoides con morfología normal, a diferencia del 63,9\% de las parejas en las que los espermatozoides con morfología normal fueron mayores al 14\%. Sin embargo, existe controversia sobre la validez de la asociación entre la integridad del componente genético de los espermatozoides y su valor predictivo, el cual ha sido especialmente cuestionado. ${ }^{9,10}$ El objetivo del presente estudio es establecer la relación entre las características espermáticas poscapacitación y la fertilización de oocitos donados y morfológicamente maduros (estadío MII) en la técnica de FIV.

\section{METODOLOGÍA}

Se realizó un estudio de corte transversal en el Centro de Biomedicina Reproductiva del Valle (Fecundar) en la ciudad de Santiago de Cali (Colombia), una institución privada que atiende pacientes de Cali y de la región suroriental de Colombia y que es centro docente para los residentes de ginecología y obstetricia de la Universidad del Valle y de la Universidad Libre que rotan por medicina reproductiva.

Las muestras seminales analizadas fueron obtenidas por medio de masturbación. Se incluyeron pacientes mayores de 18 años, normozoospérmicos, con oligozoospermia ligera (entre 10 y 20 x $10^{6}$ espermatozoides $/ \mathrm{mL}$ ) o 
moderada (entre 5 y $10 \times 10^{6}$ espermatozoides $/ \mathrm{mL}$ ) y/o astenozoospermia moderada [entre 30\% y 50\% de la motilidad progresiva total tipo $a$ (rápida) + tipo $b$ (lenta)] según los valores de referencia establecidos por la $\mathrm{OMS}^{5}$. Los óvulos donados provinieron de mujeres entre los 18 y los 30 años que forman parte del programa de donación de gametos y a quienes previamente se les realizó valoración ginecológica, psicológica, perfil hormonal, análisis cromosómico y pruebas de laboratorio para VIH, hepatitis B, hepatitis C, Chlamydia trachomatis, citomegalovirus y mononucleosis infecciosa.

Para la estimación del tamaño de la muestra se asumió una proporción esperada de fertilización óptima en el grupo de muestras con características adecuadas del 90\% y características no adecuadas del 50\%, un nivel de significancia del 5\%, un poder del 80\% y una relación de 3:1 entre adecuadas y no adecuadas. De este modo, se estimó que se requerían un total de 45 sujetos con características adecuadas según la $\mathrm{OMS}^{3}$ y 15 sujetos con características no adecuadas $(\mathrm{n}=15)$, por lo que se realizó muestreo secuencial por conveniencia.

Una vez finalizada la aspiración folicular, se procedió a eliminar los restos de granulosa con sangre de los oocitos en un medio de cultivo neutralizado. Posteriormente, se depositaron en gotas de medio de cultivo suplementadas con 10\% de albúmina sérica humana (HSA, por sus siglas en inglés; Irvine Scientific ${ }^{\circledR}$ ) en donde fueron clasificados según su madurez y se incubaron a $37^{\circ} \mathrm{C}^{11}$ con $5 \%$ de $\mathrm{CO}_{2}$. A continuación, se analizó la muestra seminal determinando el volumen total, la concentración y las motilidades espermáticas (rápidas: velocidad mayor de $25 \mu \mathrm{m} / \mathrm{seg}$, lentas: velocidad mayor de $25 \mu \mathrm{m} / \mathrm{seg}$, no progresivas e inmóviles) ${ }^{5}$ en $10 \mu \mathrm{L}$ de muestra sobre cámara Makler. Después de esto, se realizó la capacitación espermática mediante la técnica de gradientes de densidad, resuspendiendo el concentrado espermático obtenido con 300 ó $400 \mu \mathrm{L}$ de medio de cultivo en el que se determinó la concentración y la motilidad espermática. La muestra se concentró entre 20.000 y 50.000 espermatozoides móviles/mL.
Transcurridas entre 4 y 7 horas posaspiración folicular, los oocitos fueron inseminados con los espermatozoides capacitados en gotas de medio de cultivo. Después de 16 a 18 horas, los oocitos fueron decumulados para valorar la fertilización en un proceso que se evalúo por la presencia de dos pronúcleos y la extrusión del segundo cuerpo polar.

Para la realización de este estudio se consideraron las siguientes variables: edad del paciente; recuperación espermática poscapacitación; tipo de motilidad: $a$ (rápida), $b$ (lenta) y motilidad progresiva total $(a+b)$ poscapacitación; ITZ (índice de teratozoospermia) obtenido a partir de la división del número de defectos sobre el número de espermatozoides defectuosos; número de oocitos recuperados por ciclo y porcentaje de oocitos (estadío MII) fertilizados por ciclo; tipo de muestra congelada o fresca de acuerdo con la disponibilidad para el suministro de la misma; y período de abstinencia sexual.

La información se recopiló a partir de las historias clínicas de los pacientes, se consignó en una base de datos en Excel ${ }^{\circledR}$ y se analizó con el paquete estadístico Stata ${ }^{\circledR} 9 .{ }^{12}$ El análisis de información se realizó mediante la obtención de las medidas de tendencia central y de dispersión para las variables continuas y la obtención de las proporciones para las variables categóricas. La categorización de la variable fertilización se realizó tomando un valor mayor o igual a $80 \%$ como el adecuado. De igual forma, se categorizaron las variables de las características seminales de acuerdo con los valores de referencia establecidos por la $\mathrm{OMS}^{3}$ y se realizó un análisis de regresión logística en el que se relacionaron las variables de las características seminales categorizadas con la de la fertilización categorizada (0 a 80\%; $\geq 80 \%$ ), obteniéndose así un modelo que permitió determinar las variables que contribuyen a presentar mayor probabilidad de fertilización.

\section{RESULTADOS}

En este estudio se incluyeron un total de 63 pacientes con un promedio de edad de 39,9 
años (rango de 28 a 59) y se realizó análisis de rutina (precapacitación) a la muestra seminal con los siguientes hallazgos: promedio de abstinencia sexual de 4,5 días (DS $\pm 1,9$ ), volumen de 3,1 mL (DS $\pm 1,6)$, concentración espermática inicial con un promedio de 90,2 millones por $\mathrm{mL}(\mathrm{DS} \pm 67,7)$ y $47,6 \%$ en el promedio de la motilidad tipo $a+$ tipo $b$. La motilidad tipo $a$ (rápida) presentó un rango de $0 \%$ hasta 33\% con un promedio de 3,9\% (DS $\pm 6,7)$ y la tipo $b$ (lenta) presentó un promedio de 43,7\% en un rango de $7 \%$ hasta $80 \%$ (DS $\pm 15,2)$. El índice de teratozoospermia (ITZ) presentó un promedio de 1,46 (DS $\pm 0,12)$.

El análisis poscapacitación mostró que la recuperación espermática fue de 40,1 millones/mL en promedio (DS $\pm 31,2$ ), con una motilidad $a+b$ de $87,0 \%$ en promedio y un rango entre $32 \%$ y $100 \%$. La motilidad tipo a presentó un promedio de $62,5 \%$ (DS $\pm 33,1)$ y la motilidad tipo $b$ presentó un promedio de 24,4\% (DS $\pm 23,2)$. Del mismo modo, se valoró la calidad oocitaria de las donantes en las cuales la edad promedio fue de 26,2 años (rango de 20 a 36 años). El número promedio de oocitos (estadío MII) obtenidos fue de 7 , con un rango de 1 hasta 19 oocitos y un promedio de fertilización de $84,2 \%$.

Las pacientes ovoreceptoras incluidas en el estudio fueron 64, con edades comprendidas entre los 18 y los 51 años (promedio de 39,3 años).
En el presente estudio, se evidenció que el 75\% (48/64) de las muestras seminales presentaron fertilización oocitaria adecuada, es decir, mayor o igual al 80\%. No se encontraron asociaciones estadísticamente significativas al determinar la asociación entre el índice de motilidad, los días de abstinencia sexual y el índice de teratozoospermia (tabla 1).

El modelo de regresión logística mostró que la única variable que afectó la fertilización oocitaria fue la motilidad espermática tipo a poscapacitación (OR 4,33, p=0,04). La edad del paciente masculino $\geq 37$ años (OR 0,37, $\mathrm{p}=0,12)$ y el ITZ $\leq 1,59(\mathrm{OR} 3,75, \mathrm{p}=0,20)$ no presentaron una asociación estadísticamente significativa (tabla 2).

\section{DISCUSIÓN}

En este estudio se evidenció que la motilidad espermática tipo a poscapacitación superior al $25 \%$ está asociada como única variable a una mayor fertilidad. Con respecto al volumen seminal, en este estudio no se evidenció asociación de la fertilización oocitaria con el período de abstinencia sexual y el tipo de muestra (fresca o congelada).

De esta manera, en el estudio de la infertilidad masculina, el espermograma (con una sensibilidad del $86,1 \%^{13}$ ) y el análisis poscapacitación se convierten en herramientas de valor diagnóstico que

\begin{tabular}{|c|c|c|c|}
\hline Variable & Odds ratio & Valor de $\mathrm{p}$ & IC95\% \\
\hline Motilidad tipos $a+b$ poscapacitación (50\% a 100\%) & 6,71 & 0,13 & $0,56-79,66$ \\
\hline Motilidad tipo a poscapacitación (25\% a 100\%) & 3,51 & $* 0,06$ & $0,96-12,8$ \\
\hline Motilidad tipo $b$ poscapacitación ( $0 \%$ a 24\%) & 0,47 & 0,19 & $0,14-1,47$ \\
\hline Abstinencia sexual (2 a 7 días) & 1,32 & 0,64 & $0,40-4,30$ \\
\hline Índice de teratozoospermia (ITZ) $(1,0$ a 1,59$)$ & 2,09 & 0,44 & $0,31-13,8$ \\
\hline
\end{tabular}




\begin{tabular}{|l|c|c|c|}
\hline \multicolumn{4}{|c|}{ Tabla 2. Modelo de regresión logística. } \\
\hline \multicolumn{1}{|c|}{ Porcentaje } & Odds ratio & Valor de p & IC95\% \\
\hline Concentración ( $\geq 20$ millones/mL) & 3,56 & 0,31 & $0,29-43,39$ \\
\hline Motilidad tipo a poscapacitación (25\% a 100\%) & 4,33 & $* 0,04$ & $1,03-18,18$ \\
\hline ITZ (Bajo de 1 a 1,59) & 3,75 & 0,20 & $0,49-28,46$ \\
\hline Período abstinencia sexual en días (prolongado de 7 a 10) & 1,08 & 0,91 & $0,25-4,51$ \\
\hline Edad de paciente masculino ( $\geq 37$ años) & 0,37 & 0,12 & $0,10-1,30$ \\
\hline
\end{tabular}

* Valor de $\mathrm{p} \leq 0,05$

permiten evaluar las características tanto cualitativas (aspecto, licuefacción, viscosidad) como cuantitativas (volumen, $\mathrm{pH}$, concentración espermática, motilidad, morfología y vitalidad) de una muestra seminal y suministran información sobre diversas alteraciones que pueden orientar hacia las técnicas de reproducción asistida como estrategia para la obtención de un embarazo. ${ }^{14}$

La calidad espermática se mide por la cantidad, viabilidad y funcionalidad de los espermatozoides en los cuales los procesos de espermatogénesis y la capacitación son esenciales para garantizarla. ${ }^{15}$ Estudios recientes han evidenciado un continuo descenso en la calidad seminal (principalmente en la concentración) que se atribuye a factores como la polución ambiental, el tabaco, el alcohol, los pesticidas, los herbicidas y los factores ocupacionales; $;^{16-19}$ aunque también se han descubierto alteraciones en la motilidad y morfología espermática. Un estudio publicado en Europa establece que la cantidad media de espermatozoides entre los individuos de esta población ha descendido al menos en un 45\% en los últimos cincuenta años. ${ }^{20}$ En España, por ejemplo, se ha pasado de una media de 336 millones de espermatozoides por eyaculado en 1977 a 258 millones en 1995, mientras que en el Reino Unido la concentración espermática media fue de 98 millones/mL antes de 1959 y de 78 millones/mL después de 1970. En este descenso se deben tener en cuenta factores que inciden en la concentración espermática tales como la edad. El hombre produce espermatozoides durante toda su vida. No obstante, a medida que transcurre el tiempo, la cantidad y la viabilidad de las células espermáticas disminuyen. ${ }^{21}$ Por otra parte, la fertilidad se reduce lenta y progresivamente, demostrando que al menos el 50\% de los hombres mayores de 60 años conservan la calidad espermática con capacidad reproductiva. Algunos estudios incluso plantean que la fertilidad masculina puede sostenerse hasta edades cercanas a los 90 años $^{22}$, dependiendo de los estilos de vida adoptados $\mathrm{y}$ de determinados factores ambientales. ${ }^{23}$

\section{CONCLUSIÓN}

La motilidad tipo a poscapacitación superior al $25 \%$ es la variable que más se asocia con una mayor tasa de fertilidad en la técnica de fertilización in vitro.

\section{AGRADECIMIENTOS}

Agradecemos a Fecundar por permitir el acceso a la información de las historias clínicas de los pacientes incluidos en la técnica de reproducción asistida de FIV, y a todo el personal médico y del laboratorio de embriología.

\section{REFERENCIAS}

1. Practice Committee of the American Society for Reproductive Medicine. Optimal evaluation of the infertile female. Fertil Steril 2004;36:S264-7.

2. Hewitson L, Schatten G. Biología de la fecundación en la especie humana. Atlas de la reproducción asistida. Aspectos clínicos y de laboratorio. $1^{\text {a }}$ edición. Filadelfia: McGraw-Hill; 2003. p. 3-14. 
3. Ron-El R, Bracha Y, Herman A, Golan A, Soffer Y, Bukovsky I, et al. Pre-requisite work-up of the couple before in vitro fertilization. Hum Reprod 1992;7:483-6.

4. Red latinoamericana de reproducción asistida. ¿Qué es la fertilización in vitro y cuál es su eficiencia? Visitado 2008 Ago 28. Disponible en: http://www. redlara.com/esp

5. World Human Organization. WHO laboratory manual for the examination of human semen and spermcervical mucus interaction. 4th edition. Panamericana; 1999.

6. Jequier AM, Ukombe EB. Errors inherent in the performance of a routine semen analysis. Br J Urol 1993;55:434-6.

7. Pomerol JM, Arrondo JL. Práctica andrológica. Barcelona: Ediciones Científicas y Técnicas S.A.; 1994.

8. Kruger TF, Acosta AA, Simmonds KF, Swanson RJ, Matta JF, Veeck Ll, et al. New method of evaluating sperm morphology with predictive value for human in vitro fertilization. Urology 1987;30:248-51.

9. Lin MH, Kuo-Kuang Lee R, Li SH, Lu CH, Sun FJ, Hwu YM. Sperm chromatin structure assay parameters are not related to fertilization rates, embryo quality, and pregnancy rates in in vitro fertilization and intracytoplasmic sperm injection, but might be related to spontaneous abortion rates. Fertil Steril 2008;90:352-9.

10. Collins JA, Barnhart KT, Schlegel PN. Do sperm DNA integrity tests predict pregnancy with in vitro fertilization? Fertil Steril 2008;89:823-31.

11. Red Latinoamericana de Reproducción Asistida. Manual de procedimientos - Laboratorio de Reproducción Asistida; 2006.

12. Stata Corp Stata. Statistical Software: release 9.0 college Station, TX: Stata Corporation; 2005.
13. Pérez LE. Infertilidad y endocrinología reproductiva. $3^{a}$ edición. Bogotá, Colombia; 2007.

14. International Committee for Monitoring Assisted Reproductive Technology: Adamson GD, De Mouzon J, Lancaster P, Nygren KG, Sullivan E, et al. World collaborative report on in vitro fertilization, 2000. Fertil Steril 2006;85:1586-622.

15. Sloter E, Schmid TE, Marchetti F, Eskenazi B, Nath J, Wyrobek AJ. Quantitative effect of male age on sperm motion. Hum Reprod 2006;21:2868-75.

16. Perry MJ. Effect of environmental and occupational pesticide exposure on human sperm: a systematic review. Hum Reprod 2008;14:233-42.

17. Dallinga JW, Moonen EJ, Dumoulin JC, Evers JL, Geraedts JP, Kleinjans JC. Decreased human semen quality and organochlorine compounds in blood. Hum Reprod 2002;17:1973-9.

18. De Rosa M, Zarrilli S, Paesano L, Carbone U, Boggia B, Petretta M, et al. Traffic pollutants affect fertility in men. Hum Reprod 2003;18:1055-61.

19. Jensen TK, Bonde JP, Joffe M. The influence of occupational exposure on male reproductive function. Occup Med 2006;56:544-53.

20. De Kretser DM. Declining sperm counts. BMJ 1996;312:457-8.

21. Jarow JP, Espeland MA, Lipshultz Ll. Evaluation of the azoospermic patient. J Urol 2000;21:145-53.

22. Irvine S, Cawood E, Richardson D, MacDonald E, Aitken J. Evidence of deteriorating semen quality in the United Kingdom: birth cohort study in 577 men in Scotland over 11 years. BMJ 1996;312:467-71.

23. Bosh E, Reis SS, Aizpurúa J, Serra v, Lara C, Remohí J. Donación de Ovocitos. En: Remohí J, Cobo A, Romero, Pellicer A, Simón C. Manual Práctico de Esterilidad y Reproducción Humana. 2a. Edición. Madrid: McGrawHill Interamericana; 2005. p. 189-203. 